02

\title{
Флуктуирующая флуоресценция одиночных центров окраски в кристаллах фторида лития
}

\author{
(C) В.П. Дресвянский, С.А. Зилов, Е.Ф. Мартынович \\ Иркутский филиал Федерального государственного бюджетного учреждения науки Институт лазерной физики \\ Сибирского отделения РАН, \\ 664033 Иркутск, Россия \\ e-mail: nadvp@list.ru
}

Поступила в редакцию 03.08.2021 г.

В окончательной редакции 10.08.2021 г.

Принята к публикации 17.08.2021 г.

Одиночные $\mathrm{F}_{2}$ - и $\mathrm{F}_{3}^{+}$-центры окраски в кристалле $\mathrm{LiF}$ исследованы методом конфокальной флуоресцентной микроскопии с анализом и статистической обработкой временных зависимостей интенсивности их флуоресценции. Показано, что в отличие от $\mathrm{F}_{2}$-центра, который переориентируется только в триплетном состоянии, при фотовозбуждении $\mathrm{F}_{3}^{+}$-центр окраски способен переходить в триплетное состояние, однако в основном (синглетном) состоянии центр меняет ориентацию с частотой $1.5-2 \mathrm{~Hz}$ при комнатной температуре вследствие переориентационной диффузии. Также показано, что данный подвид вращательной диффузии центра не приводит к его поступательной диффузии.

Ключевые слова: флуоресценция, спектроскопия, одиночный центр окраски, конфокальная микроскопия.

DOI: $10.21883 /$ OS.2022.01.51901.34-21

\section{Введение}

Кристаллы фторида лития обладают уникальными физическими характеристиками. Они имеют простую кубическую решетку, максимальную энергию решетки, предельно широкую зону запрещенных энергий и практически не гигроскопичны. Поэтому в сравнении с другими щелочно-галоидными кристаллами (ЩГК) фторид лития имеет наиболее широкое практическое применение. В частности, он используется в качестве оптического материала для ВУФ области спектра. Широкое применение кристаллы LiF получили в термолюминесцентной дозиметрии ионизирующих излучений различных видов, включая детектирование смешанных потоков гамма- и нейтронного излучения [1]. Под действием различных источников ионизирующего излучения в кристаллах фторида лития эффективно создаются агрегатные F-, $\mathrm{F}_{2-}^{-}, \mathrm{F}_{2}^{+}-, \mathrm{F}_{3}-, \mathrm{F}_{3}^{+}-, \mathrm{F}_{4}^{-}, \mathrm{F}_{4}^{+}$-центры окраски (ЦО), а также образуются центры, ассоциированные с примесными дефектами, всегда содержащимися в кристаллах. Они являющихся рабочими квантовыми системами для создания перестраиваемых лазеров [2], пассивных лазерных затворов [3], а также в качестве объемных запоминающих оптических сред [4].

В большинстве своем ЦО представляют собой сложные квантовые системы, симметрия которых отличается от симметрии узлов кристаллической решетки. Процессы взаимодействия оптического излучения с подобными системами исследуются достаточно давно и, как правило, для ансамблей этих дефектов [5-7]. Однако из характеристик ансамбля не всегда можно получить свойства составляющих его частей. Наличие сильного электрон-фононного взаимодействия в ЩГК обусловливает однородное уширение спектральных линий, превращающихся в широкие перекрывающиеся полосы. Два типа центров могут иметь одинаковые полосы поглощения и люминесценции. Данная ситуация характерна, например для кристаллов фторида лития, в которых $\mathrm{F}_{2}$ - и $\mathrm{F}_{3}$-центры окраски имеют одну комплементарную -полосу поглощения с максимумом $441 \mathrm{~nm}$, a $\mathrm{F}_{3}^{-}$- и стабилизированные $\mathrm{F}_{2}^{+}$-центры образуют перекрывающиеся полосы люминесценции в области 800-900 nm [7]. В этом случае основным критерием различия дефектов является значение кинетического параметра - времени затухания люминесценции. Однако значения времён затухания люминесценции также могут быть плохо различимы. При изучении поведения ансамблей названных центров в этих условиях возникают проблемы, связанные с выделением вкладов отдельных типов центров. Это существенно затрудняет спектральное различие между центрами окраски и требует поиска новых принципов спектроскопии радиационных дефектов, дополняющих традиционные люминесцентные спектральнокинетические методы.

В этой связи развитие методов спектроскопии одиночных радиационных дефектов может дать новые возможности по сравнению с традиционными методами исследования ансамблей этих дефектов. Такой подход позволяет реализовать принцип познания „от простого к сложному“. Следовательно, возникает задача по развитию методов изучения одиночных радиационных дефектов в диэлектрических кристаллах. В связи с малыми размерами исследуемых объектов используемые методы должны обеспечить высокую чувствительность, 
значительное пространственное и временное разрешение. Основным экспериментальным методом исследования в данном случае является метод конфокальной сканирующей люминесцентной микроскопии, реализующий принцип время-коррелированного пространственноселективного счета одиночных фотонов, когда для каждого регистрируемого фотона определяется его время прибытия от момента возбуждения одиночного дефекта, что позволяет измерять кинетику разгорания и затухания люминесценции, определять времена жизни возбужденных состояний центров окраски и судить о вероятностях квантовых переходов в них. Данный метод люминесцентной спектроскопии высокого разрешения интенсивно развивается и уже нашел широкое применение в различных областях науки [8-11]. Анализ мировой научной литературы показывает, что ранее подобных исследований на квантовых системах, созданных радиацией в ЩГК, не проводилось. В опубликованных авторами работах [12-14] впервые методом конфокальной флуоресцентной микроскопии экспериментально наблюдалась люминесценция одиночных $\mathrm{F}_{2}$-центров в кристалле $\mathrm{LiF}$, представлены результаты анализа и статистической обработки временных траекторий интенсивности флуоресценции. Посредством компьютерного моделирования траекторий интенсивности флуоресценции одиночных $\mathrm{F}_{2}$-центров окраски в кристалле $\mathrm{LiF}$ оценена по порядку величины частота интеркомбинационных (синглеттриплетных) переходов для данных центров, получены оценки времени жизни $\mathrm{F}_{2}$-центра в первом триплетном состоянии [14]. Настоящая работа является продолжением этих исследований. Ниже методом лазерной сканирующей конфокальной люминесцентной микроскопии исследован мерцающий характер флуоресценции одиночных $\mathrm{F}_{2-}$ и $\mathrm{F}_{3}^{+}$-центров в кристаллах $\mathrm{LiF}$. B рамках проведенных исследований получен ряд интересных результатов о физических свойствах данных типов центров окраски.

\section{Описание эксперимента}

В качестве исследуемого образца использовался градиентно окрашенный $\gamma$-облученный кристалл LiF, что позволяло исследовать участки с различной концентрацией центров. На вставке к рис. 1 показан градиент концентрации, который был создан в объеме кристалла для того, чтобы была возможность найти область с концентрацией дефектов, необходимой для регистрации одиночных центров окраски. Типы центров окраски определялись по измеренным на ансамбле дефектов спектрам фотолюминесценции. На рис. 1 представлены спектры люминесценции при возбуждении непрерывным излучением светодиода с длиной волны $455 \mathrm{~nm}$, измеренные в различных областях градиентно окрашенного кристалла с помощью спектрометра OceanOptics 6500. В частности, кривые 2-4, представленные на рис. 1,

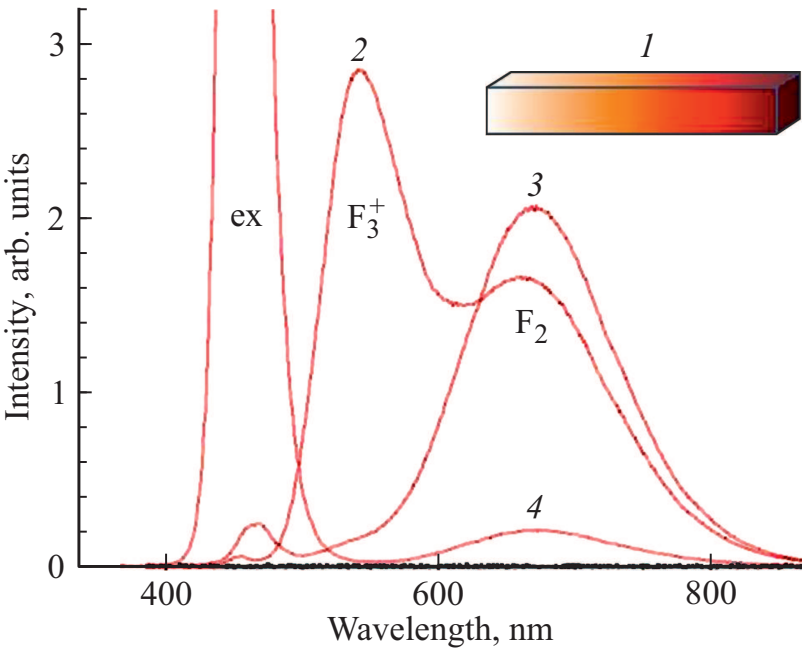

Рис. 1. Исследуемый образец LiF с градиентным пространственным распределением центров окраски (1) и спектры фотолюминесценции в правой части (2), в центральной части (3) и в левой части (4) кристалла.

указывают на присутствие в спектрах излучения полос, связанных $\mathrm{F}_{2}$ - и $\mathrm{F}_{3}^{+}$-центрами окраски.

Наблюдение люминесценции от одиночных $\mathrm{F}_{2-}$ и $\mathrm{F}_{3}^{+}$-центров в $\mathrm{LiF}$ проводилось с помощью лазерного люминесцентного конфокального сканирующего микроскопа MicroTime 200 (PicoQuant, GmbH). Для возбуждения люминесценции использовался лазер с длиной волны $470 \mathrm{~nm}$, работающий в импульсном режиме с частотой повторения импульсов 5 и $10 \mathrm{MHz}$ при длительности импульса порядка 70 ps. Возбуждающее излучение имело линейную поляризацию.

В экспериментах луч лазера был направлен по нормали к поверхности кристалла - плоскость (100) (грань куба); поворотом кристалла вокруг оси луча вектор поляризации возбуждающего излучения направлялся по одной из кристаллографических осей: либо по [100] (ориентация $0^{\circ}$, по ребру куба), либо по [110] (ориентация $45^{\circ}$, по диагонали куба). Люминесценция образца измерялась также в направлении нормали к плоскости (100).

\section{Экспериментальные результаты}

На рис. 2 представлены флуоресцентные сканированные изображения области $6 \times 6 \mu \mathrm{m}$ образца $\mathrm{LiF}$ при возбуждении пикосекундным лазером с длиной волны возбуждения $470 \mathrm{~nm}$. Программное обеспечение лазерного сканирующего конфокального флуоресцентного микроскопа MicroTime 200 формирует люминесцентные изображения дефектов с селекцией по времени затухания. Времена жизни фотолюминесценции центров двух типов, которые мы наблюдаем, различны. Светящиеся пятна на изображении представляют собой одиночные $\mathrm{F}_{2}$-центры (слева) с характерным временем затухания 


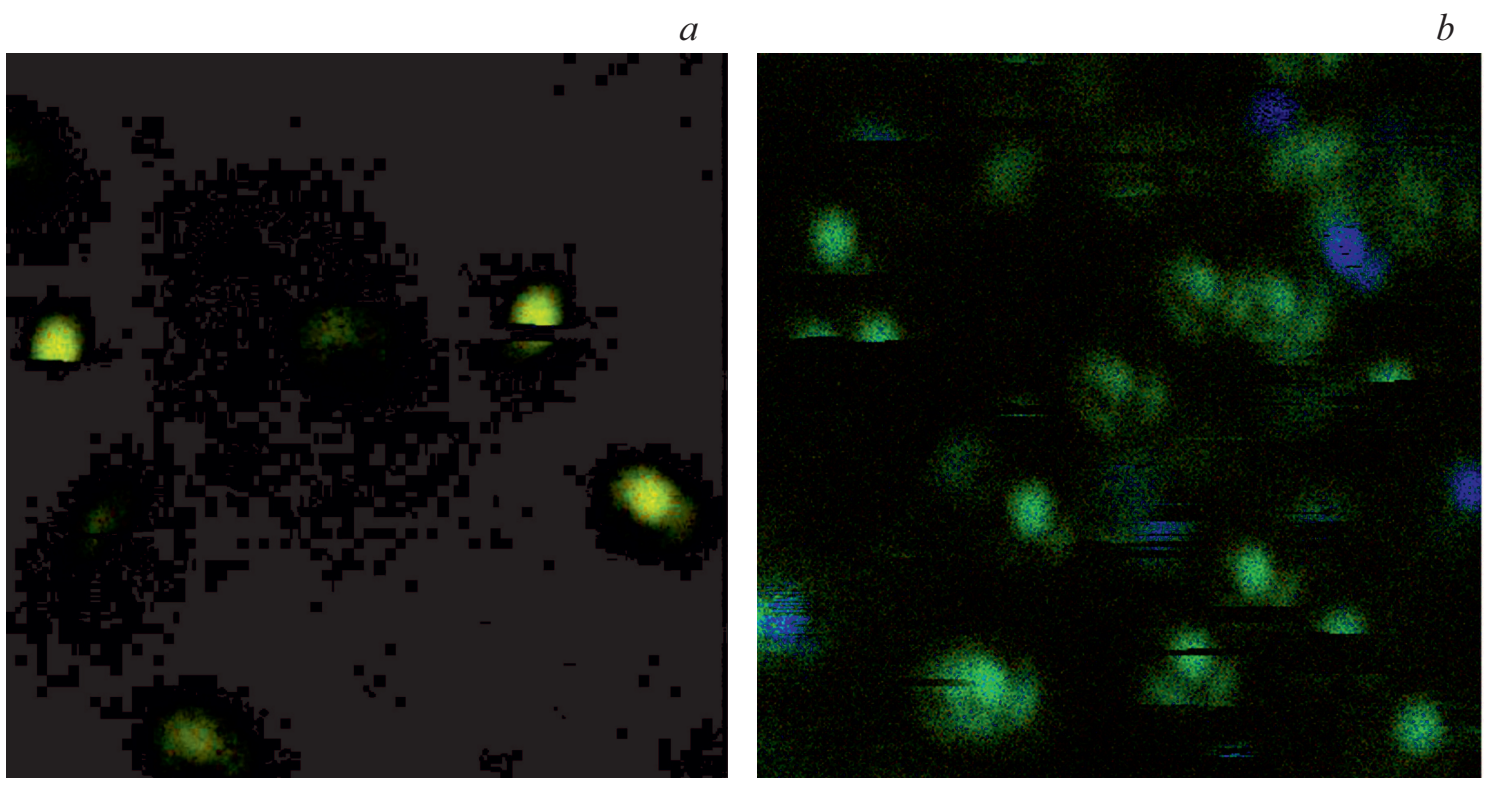

Рис. 2. Конфокальные люминесцентные изображения одиночных $\mathrm{F}_{2}-(a)$ и $\mathrm{F}_{3}^{+}$- $(b)$ центров окраски в кристалле $\mathrm{LiF}$ (область сканирования $6 \times 6 \mu \mathrm{m}$, возбуждение $470 \mathrm{~nm})$.
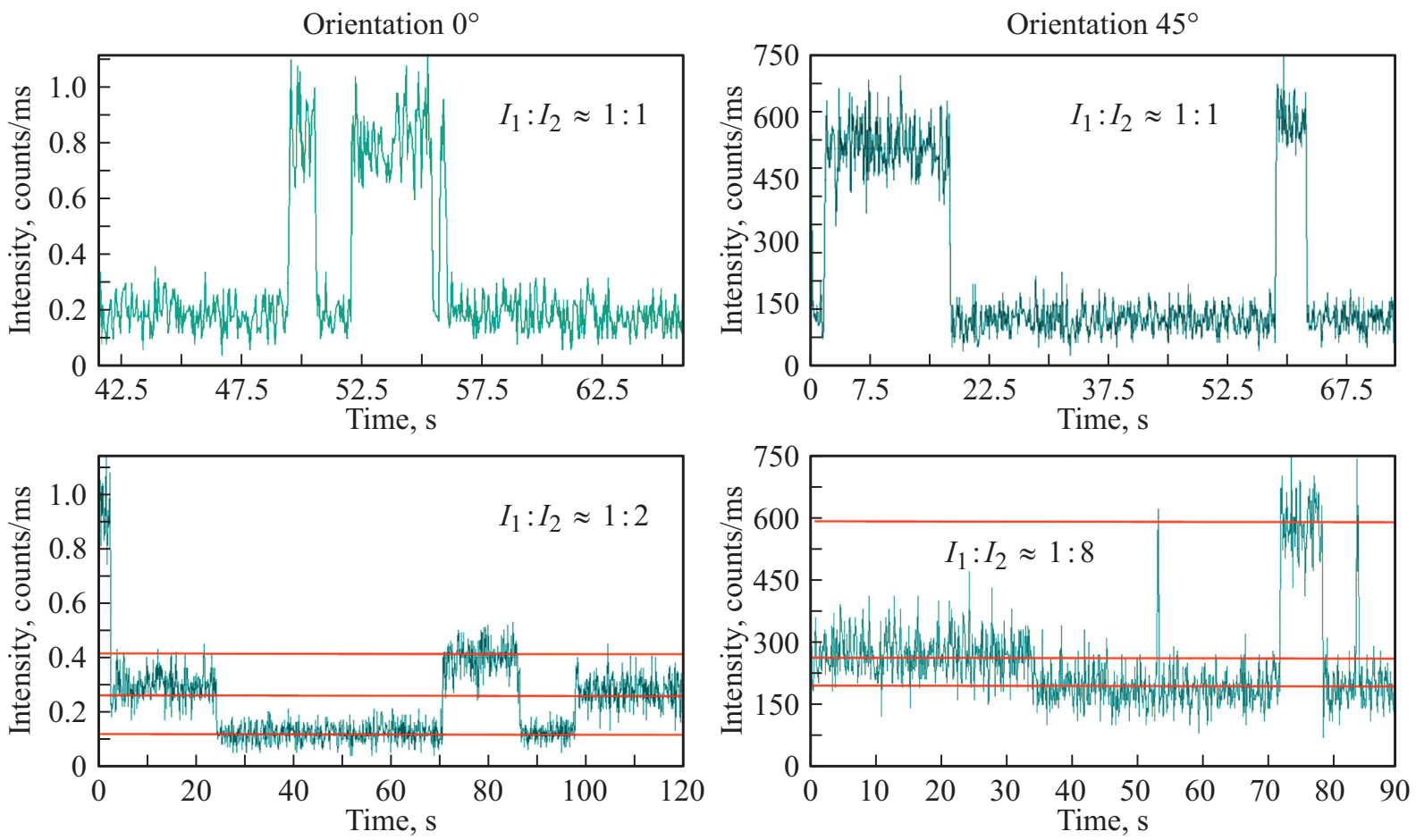

Рис. 3.

$\sim 17 \mathrm{~ns}$ и $\mathrm{F}_{3}^{+}$-центры (справа) окраски с характерным временем затухания порядка $8 \mathrm{~ns}$ [7]. Мерцающий характер изображений характерен при наблюдении флуоресценции одиночных квантовых систем. После записи люминесцентного изображения прибор наводился на одно из светящихся пятен, и записывалась зависимость интенсивности люминесценции от времени (квантовая траектория).
На рис. 3 представлены характерные квантовые траектории одиночного $\mathrm{F}_{2}$-центра при поляризации возбуждающего света вдоль ребра куба (слева) и вдоль боковой диагонали куба (справа). Интервалы времени, когда имеет место люминесценция, принято называть оn-интервалами, когда она отсутствует - off-интервалами. Резкое и полное исчезновение флуоресценции („ступенька“ на квантовой траектории) служит доказательством, что 

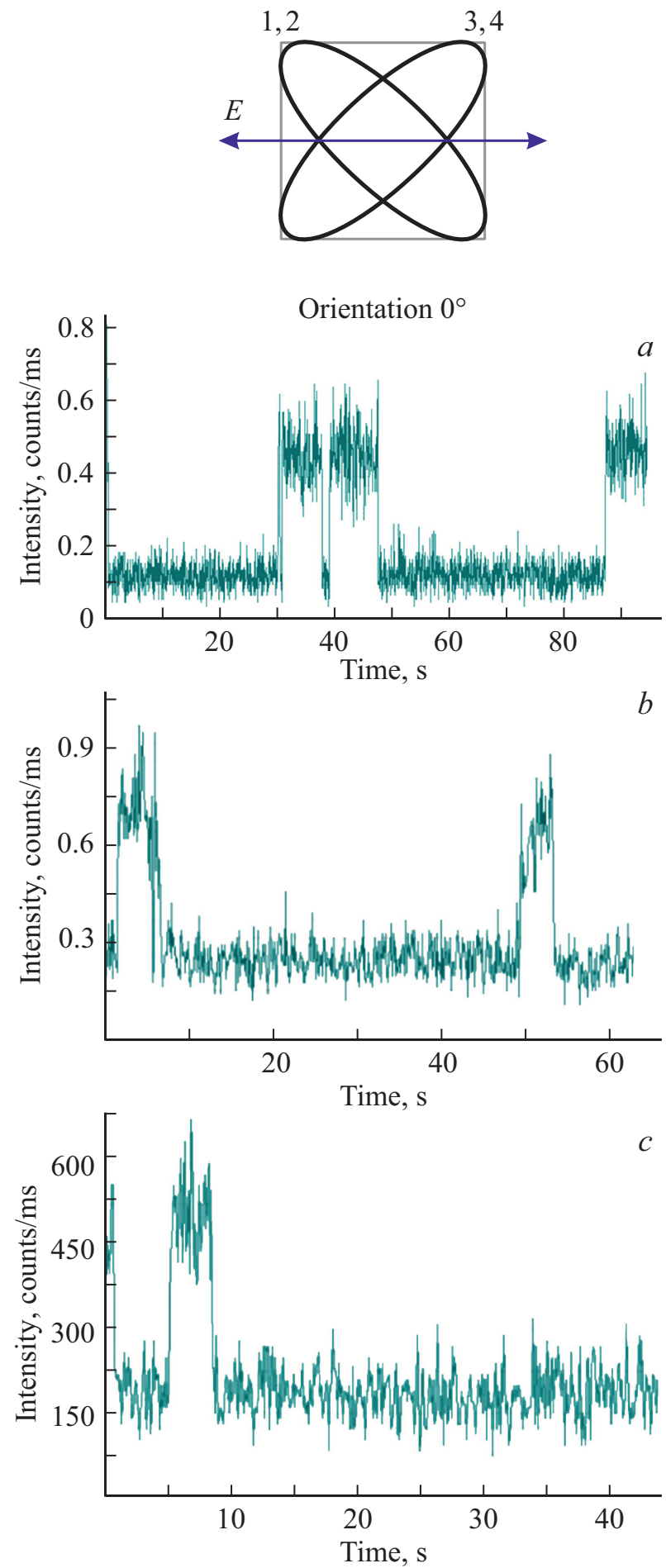

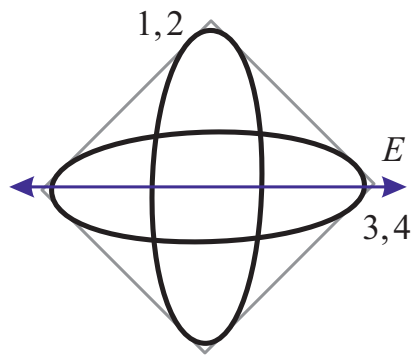

Orientation $45^{\circ}$
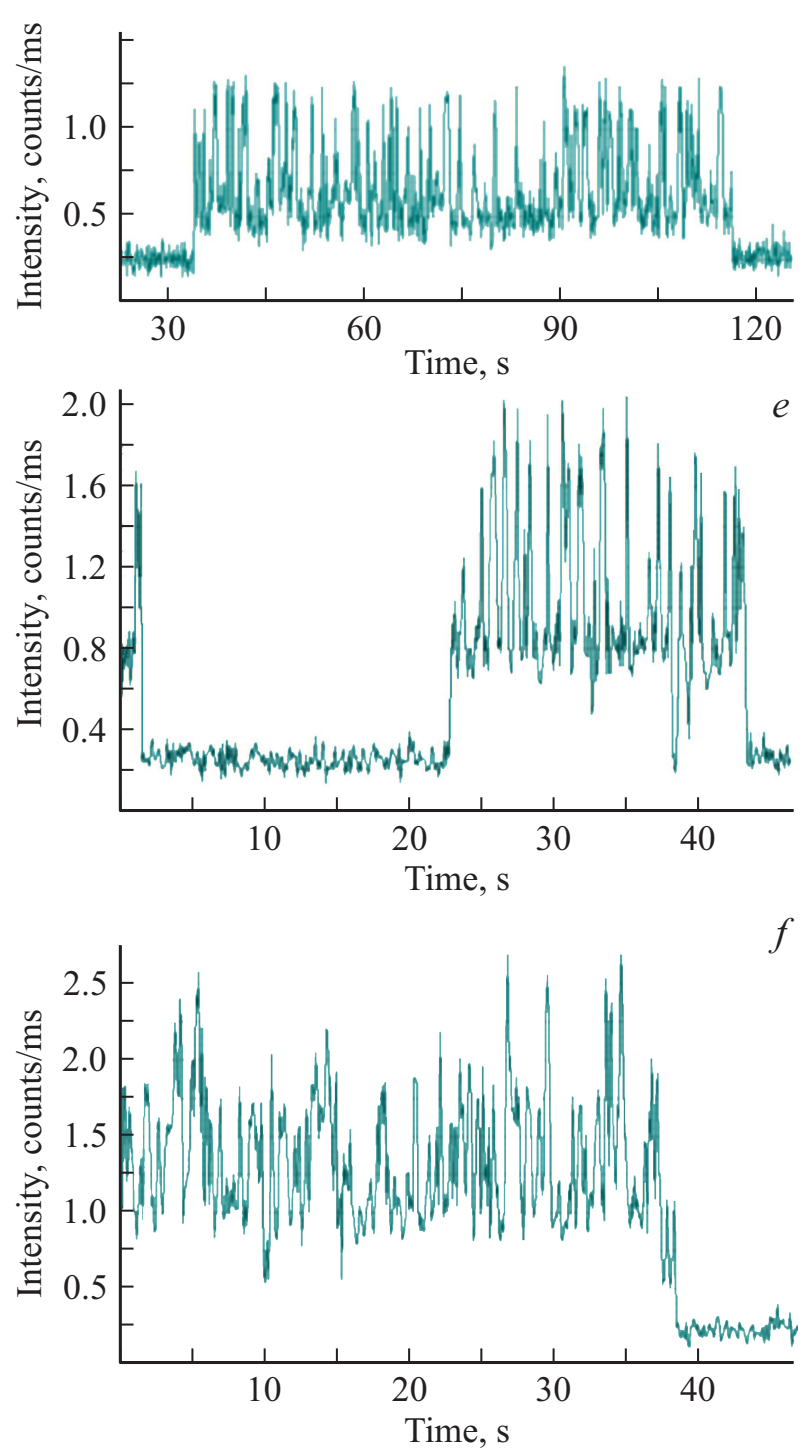

Рис. 4. Вверху показаны возможные проекции „ротаторов“ на плоскость (100) и вектор напряженности электрического поля возбуждающего света. Траектории интенсивности люминесценции одиночных $\mathrm{F}_{3}^{+}$-центров для ориентаций образца $0^{\circ}(a, b, c)$ и $45^{\circ}(d, e, f)$.

мы наблюдаем именно одиночный центр окраски. Как видим, помимо характерного для систем с триплетным уровнем чередования on и off-интервалов, наблюдаются скачки интенсивности люминесценции $I$, причем для экспериментов с ориентацией образца $0^{\circ}$ отношение интенсивностей $I_{\max } / I_{\min }$ имело значения $1: 1$ или $1: 2$, a c ориентацией образца $45^{\circ} 1: 1$ или $1: 8$.

В работе [14] авторами было показано, что это явление обусловлено переориентацией $\mathrm{F}_{2}$-центра в „темном“ триплетном состоянии. Теоретически были рассчитаны 
отношение интенсивностей люминесценции и времена жизни центра в различных ориентациях для двух конфигураций эксперимента. Полученные соотношения находятся в хорошем согласии с экспериментом.

На рис. 4 приведена небольшая серия экспериментально записанных траекторий интенсивности люминесценции одиночных $\mathrm{F}_{3}^{+}$-центров для ориентаций образца $0^{\circ}$ (рис. $3, a, b, c)$ и $45^{\circ}$ (рис. $3, d, e, f$ ).

Как видно из рис. 4, при ориентации образца $0^{\circ}$ величина флуктуаций сигнала сравнима с фоновой, а при ориентации образца $45^{\circ}$ величина флуктуаций сигнала значительно превышает фоновую, и составляет примерHo $\frac{I_{\max }}{I_{\min }}=3$.

\section{Обсуждение результатов}

Как видно из представленных на рис. 3 и 4 экспериментальных данных, зависимость интенсивности люминесценции от времени одиночного $\mathrm{F}_{3}^{+}$-центра кардинально отличается от зависимости одиночного $\mathrm{F}_{2}$-центра, однако ее характер также свидетельствует о переориентации центра при комнатной температуре.

Обнаружено, что в ходе эксперимента сигнал (интенсивность люминесценции) от центра исчезает и через некоторое время вновь появляется с той же интенсивностью. Наблюдаемое явление было интерпретировано, как переход центра с возбужденного в долгоживущее триплетное состояние с последующей релаксацией в основное состояние. В ходе экспериментов был замечен интересный факт (со 100\% повторяемостью).

1. Если возбуждающий свет падал по нормали к плоскости (100) (грань куба), а его вектор линейной поляризации был направлен по оси [100] (т. е. по одному из ребер куба), то величина флуктуации измеряемой интенсивности люминесценции $I$, в направлении той же нормали к (100), была невелика и сравнима с флуктуацией фона. В дальнейшем приведенные выше условия, будем обозначать как конфигурация эксперимента $0^{\circ}$.

2. Если же вектор линейной поляризации возбуждающего света был направлен по оси [110] (т.е. по одной из боковых диагоналей куба), то величина флуктуации измеряемой интенсивности люминесценции, в направлении той же нормали к (100), резко возрастала (конфигурация эксперимента $45^{\circ}$ ).

Для того чтобы объяснить наблюдаемое явление, напомним, что представляет собой $\mathrm{F}_{3}^{+}$-центр в щелочногалоидных кристаллах. Как известно, $\mathrm{F}_{3}^{+}$-центр представляет собой три соседние анионные вакансии (лежащие в плоскости, нормаль к которой направлена по одной из четырех осей $\mathbf{C}_{3}$ ) в решетке кристалла $\mathrm{LiF}$, захватившие пару электронов [15] (рис. 5).

Достаточно хорошей моделью $\mathrm{F}_{3}^{+}$-центра является молекулярный ион водорода $\mathrm{H}_{3}^{+}$в диэлектрической среде. Согласно теоретическим расчетам [16], молекулярный ион $\mathrm{H}_{3}^{+}$в базисе водородоподобных $1 s$-функций имеет спиновые синглетные состояния: основное, по проекции

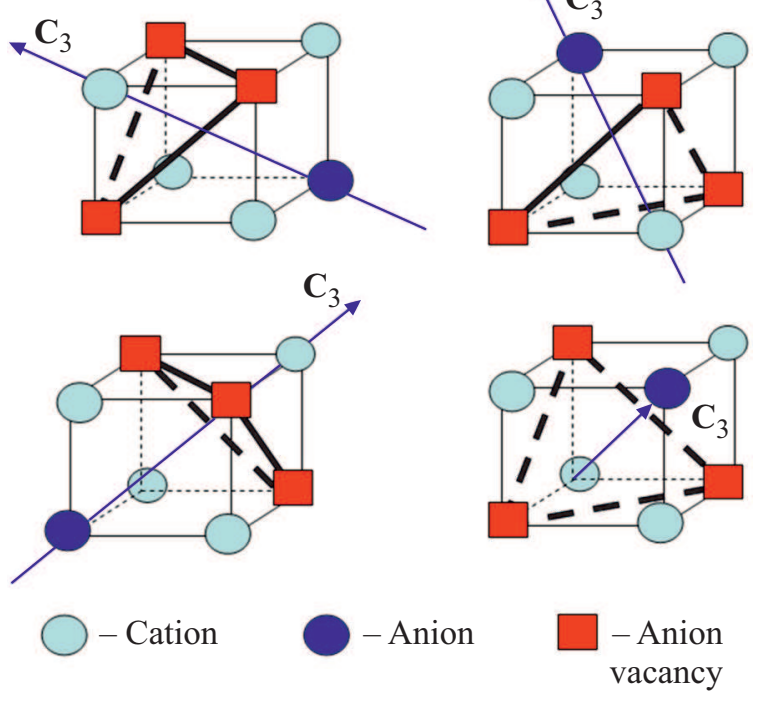

Рис. 5. Структура $\mathrm{F}_{3}^{+}$-центра и его возможные ориентации в решетке кристалла.

орбитального момента импульса невырожденное, ${ }^{1} A_{1}(1)$; два возбужденных орбитальных дуплета ${ }^{1} E_{1}(1),{ }^{1} E_{1}(2)$ и одно ${ }^{1} A_{1}(2)$-состояние, а также два спиновых триплетных терма ${ }^{3} E$ и ${ }^{3} A_{2}[17]$.

Хорошо наблюдаемые спектральные полосы поглощения $\left(\lambda_{\mathrm{abs}}^{\max }=452 \mathrm{~nm}\right)$ и люминесценции $\left(\lambda_{\mathrm{abs}}^{\max }=540 \mathrm{~nm}\right)$ $\mathrm{F}_{3}^{+}$-центра связаны с переходом ${ }^{1} A_{1}(1) \Leftrightarrow \Leftrightarrow^{1} E_{1}(1)$ [17]. Такой переход между невырожденным состоянием и дважды вырожденным по орбитальному моменту состоянием, согласно [18], описывается „ротатором“, нормаль которого ориентирована по одной из четырех возможных осей $\mathbf{C}_{3}$.

Переориентация центра означает перескок ближайшего к $\mathrm{F}_{3}^{+}$-центру аниона (показан на рис. 5) на место одной из трех вакансий. Как видно из рис. 5, переориентация $\mathrm{F}_{3}^{+}$-центра не приводит к его поступательному перемещению (что объясняет их стабильность при комнатной температуре). Данная переориентационная диффузия $\mathrm{F}_{3}^{+}$-центра обусловливает разную величину флуктуаций интенсивности люминесценции при конфигурациях эксперимента $0^{\circ}$ и $45^{\circ}$.

Как уже упоминалось, поглощение и сопряженная ему люминесценция $\mathrm{F}_{3}^{+}$-центра описывается моделью „ротаторов“. То есть поглощение и люминесценция центра описывается парой единичных взаимно перпендикулярных линейных осцилляторов $\mathbf{d}_{1}^{(i)}$ и $\mathbf{d}_{2}^{(i)}$, лежащих в плоскости, перпендикулярной одной из четырех осей $\mathbf{C}_{3}^{(i)}[9]$. Тогда нетрудно видеть, что при конфигурации эксперимента $0^{\circ}$ (рис. 4, слева) все 4 возможных ориентации центра относительно вектора $\mathbf{E}$ эквивалентны и, следовательно, флуктуации интенсивности люминесценции будут на уровне фоновых.

При конфигурации эксперимента $45^{\circ}$ (рис. 4, справа) ориентации центра 1,2 не эквивалентны ориентациям 3,4 

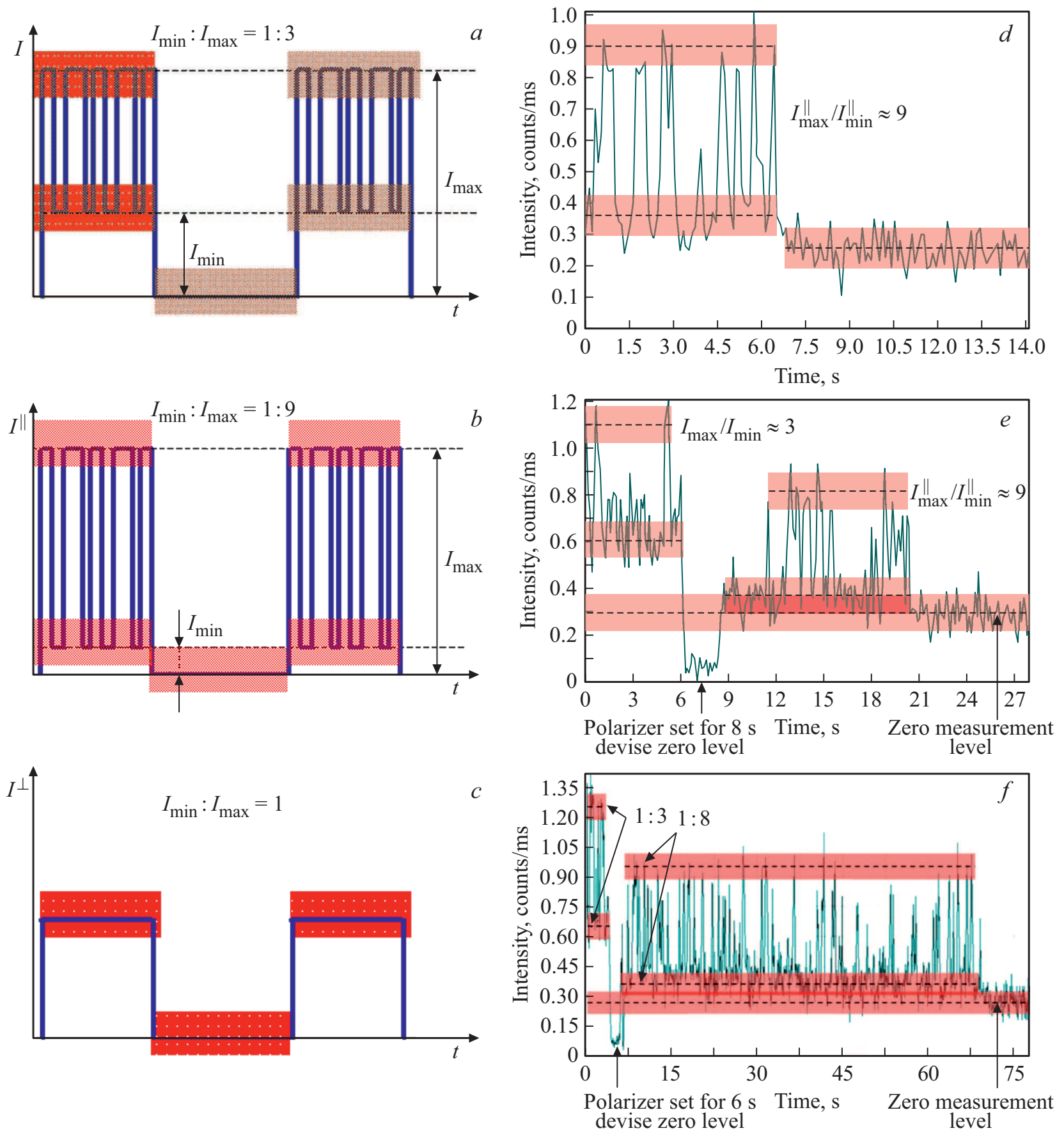

Рис. 6. Расчетные $(a, b, c)$ и измеренные $(d, e, f)$ квантовые траектории параллельной компоненты интенсивности люминесценции одиночных $\mathrm{F}_{3}^{+}$-центров для ориентаций образца $45^{\circ}$.

(относительно вектора Е). Исходя из модели „ротатора“ можно рассчитать отношение интенсивностей люминесценции одиночного $\mathrm{F}_{3}^{+}$-центра для его четырех возможных ориентаций в кристалле (рис. 5), для конфигурации эксперимента $45^{\circ}$ :

$$
\begin{gathered}
P_{1,2}^{\mathrm{absorb}}=C_{1} \cos ^{2} \alpha=C_{1}\left(\frac{1}{3}\right), P_{1,2,3,4}^{\mathrm{emit}}=C_{2}\left(1+\frac{1}{3}\right), \\
I_{1,2}=C_{1} C_{2}\left(\frac{4}{9}\right)
\end{gathered}
$$

$$
\begin{gathered}
P_{3,4}^{\text {absorb }}=C_{1}(1), \quad I_{3,4}=C_{1} C_{2}\left(\frac{4}{3}\right), \\
\frac{I_{1,2}^{\min }}{I_{3,4}^{\max }}=\frac{1}{3}, \quad \frac{\left\langle\sqrt{(\Delta I)^{2}}\right\rangle}{\langle I\rangle}=1,
\end{gathered}
$$

где $P_{i}^{\text {absorb }}, P_{i}^{\text {emit }}-$ вероятности поглощения и испускания фотона в заданном направлении за единицу времени „ротатора“ с $i$-ориентацией нормали соответственно $C_{1,2}$ - константы. 
Полученные отношения интенсивностей люминесценции для конфигурации $45^{\circ}$ находятся в качественном согласии с экспериментом (рис. 4).

Для того чтобы окончательно убедиться в правильности нашей гипотезы, были рассчитаны и измерены поляризованные компоненты интенсивности люминесценции одиночного $\mathrm{F}_{3}$-центра $I_{1,2,3,4}^{\|}$и $I_{1,2,3,4}^{\perp}$ с поляризацией параллельной и перпендикулярной поляризации возбуждающего света соответственно. Для конфигурации $0^{\circ}$ ввиду ее высокой симметрии получим тривиальный результат: $I_{1,2,3,4}^{\|}=I_{1,2,3,4}^{\perp}=\frac{1}{2} I$. Для конфигурации эксперимента , $45^{\circ \text { о6 }}$

$$
\begin{gathered}
P_{1,2}^{\text {погл }}=C_{1}\left(\frac{1}{3}\right), \quad P_{1,2}^{\text {изл. }}=C_{2}\left(\frac{1}{3}\right), \quad P_{1,2}^{\text {изл. } \perp}=C_{2}(1), \\
I_{1,2}^{\|}=C_{1} C_{2}\left(\frac{1}{9}\right), \quad I_{1,2}^{\perp}=C_{1} C_{2}\left(\frac{1}{3}\right), \\
P_{3,4}^{\text {погл }}=C_{1}(1), \quad P_{3,4}^{\text {изл. } \|}=C_{2}(1), \quad P_{3,4}^{\text {изл. } \perp}=C_{2}\left(\frac{1}{3}\right) \\
I_{3,4}^{\|}=C_{1} C_{2}(1), \quad I_{3,4}^{\perp}=C_{1} C_{2}\left(\frac{1}{3}\right) \\
\frac{I_{1,2}^{\|}}{I_{3,4}^{\|}}=\frac{1}{9}, \quad \frac{I_{1,2}^{\perp}}{I_{3,4}^{\perp}}=1 .
\end{gathered}
$$

На рис. 6 (слева) приведены результаты моделирования квантовых траекторий центра на основе расчета (4)-(6). Как видим, из расчета следует, что в случае измерения траектории компоненты люминесценции $I^{\|}$, флуктуации должны возрастать до $\frac{I_{1,2}^{\|}}{I_{3,4}^{\|}}=\frac{1}{9}$ (рис. 6, $b$ ), что и подтверждается в эксперименте (рис. 6, $d, e, f$ ). Таким образом, следует считать доказанным, что при комнатной температуре имеет место переориентационная диффузия $\mathrm{F}_{3}^{+}$-центра в основном (синглетном) состоянии.

\section{Заключение}

Следует отметить, что обнаружение описанного выше явления стало возможным благодаря применению конфокальной флуоресцентной микроскопии, которая позволяет измерять интенсивность люминесценции от одиночных квантовых систем (молекулы, центры свечения и т. п.). Традиционными методами, когда измеряется интенсивность люминесценции (и ее поляризованные компоненты) от большого числа квантовых систем, это было бы невозможно. Так как время жизни возбужденного состояния центра (порядка $8 \mathrm{~ns}$ ) много меньше среднего времени переориентации центра (около $1 \mathrm{~s}$ ), то эффект переориентации не окажет никакого заметного влияния на деполяризацию люминесценции. Также отметим, что в работе не рассматривается еще один канал ухода $\mathrm{F}_{3}^{+}$-центра в „темное“ состояние: $\mathrm{F}_{3}^{+} \Leftrightarrow \mathrm{N}_{c}$ [19].

В заключение приведем основные выводы работы.
1. Экспериментально наблюдалась переориентация одиночных $\mathrm{F}_{3}^{+}$-центров; полученные теоретически соотношения интенсивностей при различных ориентациях центра соответствуют эксперименту.

2. В отличие от $\mathrm{F}_{2}$-центра, который переориентируется только в триплетном („темном“) состоянии, $\mathrm{F}_{3}^{+}$-центр переориентируется в основном состоянии, причем такая переориентационная диффузия не приводит к поступательной диффузии центра.

\section{Благодарности}

Авторы работы выражают глубокую признательность А.Л. Ракевичу за помощь при проведении исследований на лазерном люминесцентном конфокальном сканирующем микроскопе MicroTime 200 (PicoQuant, GmbH).

\section{Финансирование работы}

Работа выполнена в рамках Плана фундаментальных исследований РАН на период до 2025 г., проект № 0243-2021-0004.

\section{Конфликт интересов}

Авторы заявляют, что у них нет конфликта интересов.

\section{Список литературы}

[1] А.И. Непомнящих, С.Н. Мироненко, Г.П. Афонин, А.И. Селявко. Атомная энергия. 58 (4), 257 (1985) [A.I. Nepomnyashchikh, S.N. Mironenko, G.P. Afonin, A.I. Selyavko. Soviet Atomic Energy. 58 (4), 292 (1985)].

[2] В.И. Барышников, В.А. Григоров, Б.Д. Лобанов, Е.Ф. Мартынович, Э.Э. Пензина, В.М. Хулугуров, В.А. Чепурной. Известия АН СССР. Серия физическая, $54(8), 1467$ (1990).

[3] Е.Ф. Мартынович, В.И. Барышников, В.А. Григоров. Письма в ЖТФ, $11(14), 875$ (1985).

[4] E.F. Martynovich, E.O. Chernova, V.P. Dresvyansky, A.E. Bugrov, P.V. Kostryukov, A.V. Konyashchenko. Optics and Laser Technology, 131, 106430 (2020). DOI: $10.1016 /$ j.optlastec.2020.106430

[5] J. Nahum. Phys. Rev., 158 (3), 814 (1967). DOI: 10.1103/PhysRev.158.814

[6] J. Nahum, D.A. Wiegard. Phys. Rev., 154 (3), 817 (1967). DOI: 10.1103/PhysRev.154.817

[7] G. Baldacchini, R.M. Montereali, T. Tsuboi. Eur. Phys. J. D., 17 (2), 261 (2001). DOI: 10.1007/s100530170030

[8] J.S. Steffen, W.E. Moerner. Current Opinion in Structural Biology, 23 (5), 778 (2013). DOI: 10.1016/j.sbi.2013.07.010

[9] E. Betzig. Rev. Mod. Phys., 87 (4), 1153 (2015). DOI: $10.1103 /$ RevModPhys.87.1153

[10] И.С. Осадько. УФН, 176, 23 (2006). DOI: $10.3367 /$ UFNr.0176.200601b.0023 [I.S. Osad'ko. Phys. Usp., 49, 19 (2006). DOI: 10.1070/PU2006v049n01ABEH002088].

[11] I.S. Osad'ko, A.V. Naumov, I.Yu. Eremchev, Yu.G. Vainer, L. Kador. Phys. Rev. A., 86 (5), 053802 (2012). DOI: $10.1103 /$ PhysRevA.86.053802 
[12] S.V. Boichenko, K. Koenig, S.A. Zilov, V.P. Dresvyanskiy, A.P. Voitovich, A.L. Rakevich, A.V. Kuznetsov, A.V. Bartul, E.F. Martynovich. J. Physics: Conference Series, $552(1)$, 012048 (2014). DOI: 10.1088/1742-6596/552/1/012048

[13] В.П. Дресвянский, С.В. Бойченко, С.А. Зилов, А.Л. Ракевич, А.П. Войтович, Е.Ф. Мартынович. Известия РАН. Серия физическая, 80 (1), 97 (2016). [V.P. Dresvyanskiy, S.V. Bojchenko, S.A. Zilov, A.L. Rakevich, E.F. Martynovich, A.P. Voitovich. Bulletin of the Russian Academy of Sciences: Physics, 80 (1), 89 (2016). DOI: $10.7868 / \mathrm{S} 0367676516010087]$

[14] С.А. Зилов, С.В. Бойченко, А.В. Кузнецов, В.П. Дресвянский, Е.Ф. Мартынович, А.Л.Ракевич, А.В. Бартуль, К. Кениг, А.П. Войтович. Известия РАН. Серия физическая, 80 (1), 89 (2016) [S.A. Zilov, S.V. Bojchenko, A.V. Kuznetsov, V.P. Dresvyanskiy, A.L. Rakevich, A.V. Bartul, K. Koenig, E.F. Martynovich, A.P. Voitovich. Bulletin of the Russian Academy of Sciences: Physics, 80 (1), 81 (2016). DOI: $10.3103 / \mathrm{S} 1062873816010251]$

[15] П.П. Феофилов. Поляризованная люминесценция атомов, молекул и кристаллов. (Гос. изд. физ.-мат. лит-ры., Москва, 1959).

[16] J.O. Hirschfelder. J. Chem. Phys., 6(12), 795 (1938). DOI: $10.1063 / 1.1750173$

[17] Т.Т. Басиев, И.В. Ермаков, К.К. Пухов. ФТТ, 39 (8), 1373 (1997). [T.T. Basiev, I.V. Ermakov, K.K. Pukhov. Physics of the Solid State, 39 (8), 1218 (1997). DOI: 10.1134/1.1130047]

[18] С.А. Зилов, Е.Ф. Мартынович. ФТТ, 50 (9), 1692 (2008) [S.A. Zilov, E.F. Martynovich. Physics of the Solid State, 50 (9), 1761 (2008). DOI: 10.1134/S1063783408090333].

[19] В.М. Костюков, Н.Т. Максимова, З.И. Мыреева, С.А. Зилов. Опт. и спектр., 79 (4), 625 (1995). 